\title{
Diffuse Large B-Cell Lymphoma Mimicking Schwannoma of Lumbar Spine
}

\author{
Seung-Kook Kim, Sun-Ho Lee, Eun-Sang Kim, Whan Eoh \\ Department of Neurosurgery, Spine Center, Samsung Medical Center, Sungkyunkwan University School of Medicine, Seoul, Korea
}

\begin{abstract}
A rare case of solitary diffuse large B-cell lymphoma arising from the lumbar spinal nerve root is reported. A 37-year-old man presented with a 3-month history of progressive numbness and paraparesis in both legs. The initial diagnosis was benign primary intradural extramedullary tumor including schwannoma and meningioma. Histopathological examination revealed diffuse large B-cell lymphoma. While a well-defined T1 isointense mass is common in primary spinal schwannoma, the present case was atypical and had a yellowish neural component. The pathogenesis and radiological findings of spinal diffuse large B-cell lymphoma are discussed and related literature is reviewed.
\end{abstract}

Key Words: Diffuse large B-cell lymphoma $\cdot$ Extramedullary spinal cord compression $\cdot$ Schwannoma

\section{INTRODUCTION}

Non-Hodgkin's lymphoma (NHL) is diagnosed in approximately 31,700 individuals each year in the United States. NHL affects the nervous system either primarily or by metastatic involvement ${ }^{8)}$. Spinal compression due to diffuse large B-cell lymphoma (DLBL) is reported in $0.1 \%$ to $10.2 \%$ of patients with $\mathrm{NHL}^{7}$. Considering the incidence and clinical features, a well-defined intradural extramedullary tumor including schwannoma or meningioma is suspected. But in rare cases, lymphoma can result in misdiagnosis and flawed surgical strategy. In the context of this case report, we discuss the characteristics of a spinal DLBL on magnetic resonance imaging (MRI), predisposing medical conditions that should cause the neurosurgeon to add lymphoma to the normal differential diagnosis, and the choice of appropriate management.

\section{CASE REPORT}

A 37-year-old man presented with a 3-month history of progressive numbness and paraparesis in both legs. Upon admission, the patient presented with predominant paraparesis and left arm numbness. Three months previously he had experienced the first onset of leg numbness and weakness. He had visited local clinic and received a MRI scan. He was referred

- Received: November 3, 2015 • Revised: January 17, 2016

- Accepted: January 18, 2016

Corresponding Author: Whan Eoh

Department of Neurosurgery, Samsung Medical Center, Sungkyunkwan University School of Medicine, 81 Irwon-ro, Gangnam-gu, Seoul 06351, Korea

Tel: +82-2-3410-3499, Fax: +82-2-3410-0048

E-mail: w.eoh@samsung.com

$\otimes$ This is an open access article distributed under the terms of the Creative Commons Attribution Non-Commercial License (http://creativecommons.org/licenses/by-nc/4.0/) which permits unrestricted non-commercial use, distribution, and reproduction in any medium, provided the original work is properly cited. to Spine Center of Samsung Medical Center for further evaluation. A neurologic examination revealed decreased big toe power (grade IV). MRI revealed a $2.0-\mathrm{cm}$ intradural extramedullary mass. T2-weighted imaging (T2WI) showed slightly low-signal intensity (Fig. 1A). Short time inversion recovery (STIR) contrast enhanced images showed an enhanced, well-defined mass at the L3 level (Fig. 1B). The initial diagnosis was primary spinal benign tumor including schwannoma or meningioma. Partial hemilaminectomy of the L3 lower portion and L4 upper lesion through a right unilateral approach was done. In the microscopic operation field, we confirmed a relatively well-defined oval-shaped mass with 3 connections with the dural area. After confirming normal motor evoke potential (MEP), the three connections were severed and the mass was removed en block. A spindle-like mass was evident. This mass was somewhat yellower than the main mass. That mass was also removed after confirming MEP. After removal of the 2 masses, the neural element changed to a yellow color. Residual lesions could not be removed because of the risk of permanent neurologic deficits. Postoperatively, symptoms disappeared but left shoulder pain was evident. Brachial plexus MRI scans were taken. The
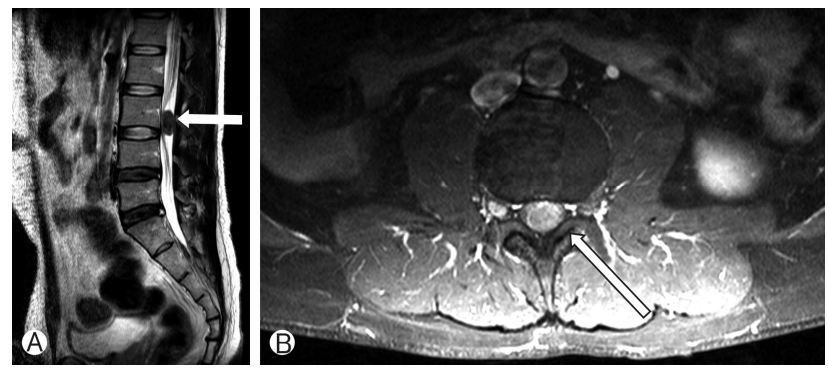

Fig. 1. (A) Preoperative T2-weighted image showing well defined low intensity mass lesion in L3 level (white arrow). (B) Preoperative short time inversion recovery with contrast enhancement image shows an intradural mass at the marked enhancement with contrast (white arrow). 


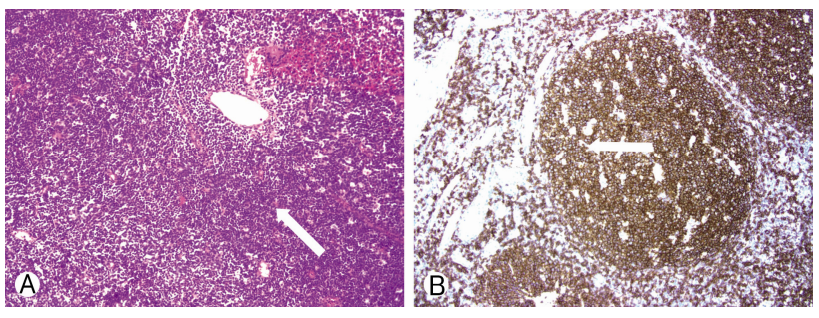

Fig. 2. (A) Diffuse proliferation of lymphoid cells in the mass (white arrow). There was no neural involvement. (B) Positivity of tumor cell to CD20, characterizing B-cell lymphoma (white arrow).

brachial plexus scan revealed left C7 and C8 nerve preganglionic hyperintensity, reflecting the origin of neuropathy at these levels. Histopathological examination revealed DLBL (Fig. 2A). Immunohistochemistry for CD20 and bcl-2 were positive. Bcl-6 and MUM-1 showed 70\% positivity and Ki-67 each showed 85\% positivity for tumor cells (Fig. 2B). In situ hybridization for Epstein-Barr virus was negative. A postoperative positron emission tomography scan revealed focal uptake of fluorodeoxyglucose in the sacral canal that suggested malignant lymphoma involvement. The patient was transferred to the Oncology Department for methotrexate based chemotherapy. After chemotherapy, he was discharged without complications.

\section{DISCUSSION}

The present case clinically represents DLBL with initial manifestation of both leg numbness and paraparesis of the lower extremities. Intradudal spinal schwannomas and meningiomas are the most common tumors, which cause progressive neurological symptoms. These 2 lesions account for $45 \%$ of primary spinal neoplasms ${ }^{2}$. Considering the incidence, a well-defined thoraco-lumbar mass is usually regarded as a primary spinal tumor $^{2)}$. Based on this reasoning, we initially chose surgical remo val.

Treatment of each tumor is different. Schwannoma is a slowgrowing benign tumor, in which the adjacent normal tissue is relatively tolerant to chronic compression of mass, and functional recovery of neural tissue after total removal of the tumor can be expected. Therefore, in a case in which the tumor is readily accessible through a posterior approach, the neurosurgeon should choose laminectomy, with a dural incision and gross total resection as the treatment of choice. Many authors have reported high success rates (85\%-97.2\%) for gross total resection of spinal intradural extramedullary tumors ${ }^{3,4,6}$. One study reported that $80 \%$ of the lesions showed well-demarcated dissection plane and no adhesion to the spinal cord, with gross total resection being easily achieved, which was similar to the reported gross total resection rate of $70 \%-90 \%$ for intramedullary tumors ${ }^{13)}$. Another study reported recurrence rates of $10.7 \%$ after 5 years and $28.2 \%$ after 10 years of follow-up, and concluded that partial removal, recurrent tumor surgery, neurofibromatosis type 2 , and old age predis- pose for tumor recurrence. In one study, 50\% of subtotally removed tumors showed clinical recurrence ${ }^{11)}$. In a study of 20 patients in whom subtotal resection was performed, recurrence occurred in $55 \%$ of the cases ${ }^{11)}$. Convertsely, recurrence in another study involved only patients treated by gross total resection, with none of the subtotal resections experiencing tumor recurrence. In the present case, the surgeon may have overlooked a small piece of tumor, which subsequently fueled recurrence. This possibility is typically minimized by en bloc tumor removal, with schwannoma and meningioma treated by gross total resection.

Definitive diagnosis of primary spinal lymphoma may require open biopsy, although collection of cerebrospinal fluid (CSF) for diagnosis has been advocated ${ }^{12}$. Presently, we did not obtain CSF before surgery. If testing of CSF identifies a systemic lymphoma, open biopsy is not chosen initially to reduce the risk of paraplegia after surgery. Primary central nervous system lymphoma (PCNSL) is best treated using combined chemotherapy rather than radiotherapy or chemotherapy alone ${ }^{1)}$. Methotrexate is the single most effective chemotherapeutic agent for PCNSL ${ }^{1,9)}$. Radiation therapy alone is initially effective but the response is usually short-lived with median survival rates ranging from 10 to 18 months ${ }^{1)}$. A dose exceeding $>50 \mathrm{~Gy}$ and the addition of a boost are associated with increased risk of neurotoxicity ${ }^{9}$. Aggressive surgical tumor resection is not effective for the treatment of PCNSL lesions ${ }^{1)}$.

Spinal intradural extramedullary tumors simultaneously display different signals and enhanced features, which can allow a more reliable qualitative diagnosis. Schwann cell neurilemmo$\mathrm{ma}$ and neurofibroma originate from the nerve sheath, and are often referred to as neurogenic tumors. Schwannomas and neurofibromas are located in any spinal canal segment, mainly at the cervical and the thoracic segment, with some lumbar involvement. MRI signals of neurilemmoma and neurofibroma are similar and typically show equal or low signals in T1weighted imaging (T1WI) and high signal in T2WI. Enhanced scans reveal homogeneous or inhomogeneous enhancement. Meningioma is usually located in the cervical and upper thoracic segments, with the lumbosacral segment rarely involved. Most meningiomas have a longitudinal diameter $<2.5 \mathrm{~cm}$, a transverse diameter $<1.5 \mathrm{~cm}$, and no foraminal extension ${ }^{10)}$. Meningioma signals are relatively homogenous; T1WI imaging has an equal or low signal, and T2WI imaging has a low or slightly high signal. Enhancement scanning of the meningioma reveals a heterogeneous enhancement, and the strengthening degree is lower than in neurogenic tumors ${ }^{10)}$. In addition, the dural tail sign aids in the diagnosis of meningioma. Several neurofibromas reportedly featured the dural tail sign ${ }^{11)}$, implying that this sign is not the peculiar MRI feature of meningioma. Lymphoma is not limited to a single spinal compartment. Spinal cord involvement usually reflects metastatic disease. PCNSL is uncommon in the spinal cord. PCNSL demonstrates solid enhancement with adjacent high T2 signal intensity, consistent with edema. Cord enlargement is not as 
severe as with other intramedullary neoplasms ${ }^{5}$. Primary dural lymphoma presents with diffuse enhancement and can be confused with meningioma.

In our case, the mass was located at the L3 level. Intensity of T1WI and T2W1 image was intermediate and low, respectively. This implies that cellular components are more compact than benign spine neoplasm. In the STIR imaging, enhancement can be achieved for a highly vascular lesion including malignant neoplasms, such as lymphomas. Even if a single, well-defined mass in the lumbar lesion is the only apparent neurologic symptom, malignant lesion, such as a primary lymphoma, should be considered. If the mass has a possibility of being a spinal benign neoplasm and malignant neoplasm, CSF cytology should be considered before surgery.

\section{CONCLUSION}

Primary DLBL is a rare neoplasm in the spine. It is usually evident as multiple lesions consistent with edema. But, these tumors have higher cellular density that can be differentiated in a MRI scan. Primary benign spinal neoplasm and DLBL require different surgical strategies and treatment. A neurosurgeon must consider the possibility of malignant neoplasm when confronted by a single spinal mass.

\section{CONFUCT OF INTEREST}

No potential conflict of interest relevant to this article was reported.

\section{REFERENCES}

1. Abrey LE, Yahalom J, DeAngelis LM: Treatment for primary CNS lymphoma: the next step. J Clin Oncol 18:3144-3150, 2000
2. Abul-Kasim K, Thurnher MM, McKeever P, Sundgren PC: Intradural spinal tumors: current classification and MRI features. Neuroradiology 50:301-314, 2008

3. Albanese V, Platania N: Spinal intradural extramedullary tumors: personal experience. J Neurosurg Sci 46:18-24, 2002

4. Cho JH, Cho DC, Sung JK, Kim KT: Primary malignant lymphoma in a spinal cord presenting as an epidural mass with myelopathy: a case report. Korean J Spine 9:265-268, 2012

5. Chung JY, Lee JJ, Kim HJ, Seo HY: Characterization of magnetic resonance images for spinal cord tumors. Asian Spine J 2:15-21, 2008

6. Conti P, Pansini G, Mouchaty H, Capuano C, Conti R: Spinal neurinomas: retrospective analysis and long-term outcome of 179 consecutively operated cases and review of the literature. Surg Neurol 61:34-43, 2004

7. Epelbaum R, Haim N, Ben-Shahar M, Ben-Arie Y, Feinsod M, Cohen Y: Non-Hodgkin's lymphoma presenting with spinal epidural involvement. Cancer 58:2120-4, 1986

8. Ersbøll J, Schultz HB, Thomsen BL, Keiding N, Nissen NI: Meningeal involvement in non-Hodgkin's lymphoma: symptoms, incidence, risk factors and treatment. Scand J Haematol 35:487496, 1985

9. Ferreri AJ: How I treat primary CNS lymphoma. Blood 118: 510-522, 2011

10. Gu R, Liu JB, Zhang Q, Liu GY, Zhu QS: MRI diagnosis of intradural extramedullary tumors. J Cancer Res Ther 10:927931, 2014

11. Kutcher TJ, Brown DC, Maurer PK, Ghaed VN: Dural tail adjacent to acoustic neuroma: MR features. J Comput Assist Tomogr 15:669-670, 1991

12. MacKintosh FR, Colby TV, Podolsky WJ, Burke JS, Hoppe RT, Rosenfelt FP, et al: Central nervous system involvement in nonHodgkin's lymphoma: an analysis of 105 cases. Cancer 49:586595, 1982

13. Nakamura M, Ishii K, Watanabe K, Tsuji T, Takaishi H, Matsumoto $\mathrm{M}$, et al: Surgical treatment of intramedullary spinal cord tumors: prognosis and complications. Spinal Cord 46:282-286, 2008 